\title{
Chemical composition of hyssop cv. "Domaći ljubičasti" essential oil and its antimicrobial activity
}

\author{
Milica Aćimović 1 Ana Varga ${ }^{2}$ Mirjana Cvetković 3 Pezo Lato PBiljana Lončar $^{5}$. \\ Maja Ignjatov ${ }^{1} \cdot$ Tijana Zeremski $^{1}$
}

${ }^{1}$ Institute of Field and Vegetable Crops Novi Sad, Maksima Gorkog 30, 21000 Novi Sad, Serbia

2University of Novi Sad, Institute of Food Technologies, Bulevar cara Lazara 1, 21000 Novi Sad, Serbia

${ }^{3}$ University of Belgrade, Institute of Chemistry, Technology and Metallurgy, Njegoševa 12, 11000 Belgrade, Serbia

${ }^{4}$ University of Belgrade, Institute of General and Physical Chemistry, Studentski trg 12-16, 11000 Belgrade, Serbia

${ }^{5}$ University of Novi Sad, Faculty of Technology, Bulevar cara Lazara 1, 21000 Novi Sad, Serbia

\begin{abstract}
Summary: Hyssop (Hyssopus officinalis L., Lamiaceae) is a perennial shrub or subshrub violet-blue flowers in verticillasters and spicy taste with a pungent flavour. Besides being used as a culinary herb for flavouring and food preservation, this plant is also an ornamental, bee attracting plant and a traditional remedy for respiratory diseases and digestive disturbances. Hyssop is an essential oil-bearing plant, and its essential oil (Hyssopi aetheroleum) is used in the pharmaceutical, perfume and cosmetics industries as well as in aromatherapy. The objective of this study was to determine the chemical composition of essential oil of hyssop cv. "Domaći ljubičasti", grown in Serbia, and investigate its antimicrobial activity against 16 bacteria, mainly pathogens in the food industry. A total of 61 compounds were detected in the hyssop essential oil. The bicyclic monoterpene ketones cis-pinocamphone (43.8\%) and transpinocamphone $(18.3 \%)$ were the most abundant, comprising $62.1 \%$, followed by $\beta$-pinene $(6.3 \%)$ and pinocarvone (6.1\%). Hyssop essential oil expressed antibacterial activity against: Staphylococcus aureus, Escherichia coli, Bacillus cereus, Proteus hauseri, Listeria monocytogenes, Rhodococcus equi, Listeria ivanovii, Salmonella Enteritidis, Enterococcus faecalis, Listeria innocua and Bacillus spizizenii. Hyssop essential oil did not express antibacterial activity against Pseudomonas aeruginosa, Salmonella Typhimurium, Klebsiella aerogenes and Staphylococcus epidermidis. Results of this study show that hyssop essentia oil has potential for using as natural supplement for control of foodborne diseases of microbiological origin, as well as flavor compositions (herbaceous, camphor-like odour with warm and spicy undernotes), especially for meat products, sauces, soups and seasonings.

Key words: antibacterial activity, essential oils, hyssop, Hyssopus officinalis, pinocamphone
\end{abstract}

\section{Introduction}

Hyssop (Hyssopus officinalis L., Lamiaceae) is a polymorphous self-fertile plant species that grows as a perennial shrub or subshrub, up to $20-60 \mathrm{~cm}$ high. The root is strong branching, multi-headed and taproot. Stems are straight, woody and branched at the base. Leaves are opposite to each other, lanceolate, stalkless

\section{Corresponding author:}

milica.acimovic@ifvens.ns.ac.rs

Acknowledgements:

This research was supported by the Ministry of Education, Science and Technological Development of the Republic of Serbia, grant number: 451-039/2021-14/200032.

Cite this article:

Aćimović M., Varga A., Cvetković M., Pezo L., Lončar B., Ignjatov M., Zeremski T. (2021). Chemical composition of hyssop cv. "Domaći ljubičasti" essential oil and its antimicrobial activity. Ratar. Povrt., 58 (1), 23-30.

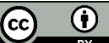

Copyright (C) The Author(s) 2021 and toothless, dark green, covered with thin silky hairs, and bearing essential oil-producing glands. Flowers are hermaphrodite, tubular, two-lipped, clustered in upper leaf axils, usually 3-9 in the nodes, often forming a spike. Fruit is small (up to $2.5 \mathrm{~mm}$ in length), oblong trihedral achene, and dark brown (Judžentiene, 2016, Aćimović et al., 2019a).

Hyssop has quite a spicy taste with a pungent flavour (Jahantigh et al., 2016). Because of this, it is used as a culinary herb for flavouring and food preservation (Moro et al., 2011) of meat products, sauces, soups and seasonings, alcoholic beverages, bitters and liqueurs (Kizil et al., 2008, Jahantigh et al., 2016, Ozer et al., 2005, Baj et al., 2018). It is also an ornamental and bee attracting plant (Piccaglia et al., 1999). In Serbian folk medicine, hyssop is used in case of respiratory diseases (for chronic bronchitis and asthma relief) and digestive disturbances as a part of tea blends (Tucakov, 2006). The same use is also present in other traditional medicines such as Bulgarian (Nanova et al., 2007), 
Turkish (Said-Al Ahl et al., 2015), as well as Unani and Ayurveda (Fathiazad and Hamedeyazdan, 2011, Tahir et al., 2018). However, hyssop is mostly used for essential oil distillation. This essential oil is used in the pharmaceutical and perfume industries (perfumes, especially in ean de cologne and oriental bases) and cosmetics (for soaps) as well as in aromatherapy (Jahantigh et al., 2016, Judžentiene, 2016, Aćimović 2021).

Only $H$. officinalis ssp. aristatus grows wild on sunny and calcareous hillsides in Serbia as well as in Eastern Europe (Josifović, 1975, Piccaglia et al., 1999). Selected hyssop cultivars $H$. officinalis ssp. officinalis are commercially cultivated in France, Italy, Germany, Serbia, Montenegro, Slovenia, Croatia, Bosnia and Herzegovina, Bulgaria, Hungary, Holland, Poland, Moldova, Iran, China, India, Russia and USA (Gorunović et al., 1995, Kizil et al., 2008, Fraternale et al., 2004, Judžentiene, 2016, Zawislak, 2013). Several cultivars are being grown: Sophie, Erfurter Ysop, Blankyt, Hysop lekarsky, Cyrano (Nemeth-Zambori et al., 2017). The crop can be productive for approximately 10 years and the plants are harvested preferably during the blooming season to produce essential oil by steam distillation (Hamida et al., 2020).

The objective of this study was to determine the chemical composition of essential oil of hyssop cv. "Domaći ljubičasti", grown in Serbia, was used to investigate its antimicrobial activity against 16 bacteria, mainly pathogens in the food industry.

\section{Material and Methods}

\section{Plant material}

The commercial hyssop variety "Domaći ljubičasti“ (H. officinalis ssp. officinalis $\mathrm{f}$. cyaneus) were grown at the Institute of Field and Vegetable Crops Novi Sad, during 2019. During the flowering stage (July), the aboveground parts were cut, dried and used for essential oil extraction.

\section{Essential oil extraction}

The dried aboveground parts of hyssop (Hyssopi herba) were subjected to hydro-distillation using an allglass Clevenger-type apparatus to extract essential oils (Hyssopi aetheroleum). The samples were ground, homogenized and made into a fine powder. To extract the essential oils, $100 \mathrm{~g}$ of the powder was placed in $1 \mathrm{l}$ conical flask and connected to the Clevenger apparatus. $500 \mathrm{ml}$ of distilled water was added to the flask and heated to the boiling point. The steam combined with the essential oils was distilled into a graduated cylinder for $4 \mathrm{~h}$ and then separated from the aqueous layer. The essential oil amount was meagre, and therefore it was extracted with n-hexane, dried over anhydrous sodium sulfate and evaporated. The obtained oil was kept refrigerated at $+4{ }^{\circ} \mathrm{C}$ until required for further analysis.

\section{GC/FID and GC/MS analysis}

The essential oil composition was determined by GC/FID and GC/MS. The GC analysis was performed on Agilent 6890N GC system equipped with 5975 MSD and FID, using HP-5 MS column $(30 \mathrm{~m} \times 0.25 \mathrm{~mm}$, $0.25 \mu \mathrm{m}$ film thickness). The injection volume was $2 \mu \mathrm{L}$ and the injector temperature was $200{ }^{\circ} \mathrm{C}$ with a $10: 1$ split ratio. Helium was the carrier gas and its flow rate was $1.0 \mathrm{~mL} / \mathrm{min}$ (constant flow mode). The column temperature was linearly programmed in the range 60 $280^{\circ} \mathrm{C}$ at a rate of $3^{\circ} / \mathrm{min}$ and held at $280{ }^{\circ} \mathrm{C}$ for $5 \mathrm{~min}$. The transfer line was heated at $250{ }^{\circ} \mathrm{C}$. The FID detector temperature was $300{ }^{\circ} \mathrm{C}$. EI mass spectra $(70$ $\mathrm{eV}$ ) were acquired in the $\mathrm{m} / \mathrm{z}$ range $35-550$. The retention indices were experimentally determined using n-alkanes (C8-C20 and C21-C40) injected under the same chromatographic conditions. The identification of the compounds was based on the comparison of their retention indices (RI), their retention times (tR) and mass spectra with those obtained from authentic samples and/or the NIST AMDIS (Automated Mass Spectral Deconvolution and Identification System) software, Wiley libraries, Adams database and literature.13 Relative percentages of the identified compounds were computed from the GC/FID peak area

\section{Antimicrobial activity}

The antimicrobial activity of the tested sample was evaluated by using 16 strains from American Type Culture Collection. There were seven tested Gramnegative bacteria in this investigation: E. coli (ATCC 8739 and ATCC 10536), K. aerogenes (ATCC 13048), P. bauseri (ATCC 13315), P. aeruginosa (ATCC 27853), S. Enteritidis (ATCC 13076) and S. Typhimurium (ATCC 14028). There were nine tested Gram-positive bacteria: B. cereus (ATCC 11778), B. spizizenii (ATCC 6633), E. faecalis (ATCC 29212), L. innocua (ATCC 33090), L. ivanovii (ATCC 19119), L. monocytogenes (ATCC 19111), R. equi (ATCC 6939), S. aureus (ATCC 25923) and S. epidermidis (ATCC 12228).

According to the National Committee for Clinical Laboratory Standards, the antimicrobial activity of hyssop essential oil was tested by the modified broth microdilution method (NCCLS 2002). The microbial strains suspensions were prepared from overnight broth cultures and were adjusted to $0.5 \mathrm{McF}$ arland standard turbidity (corresponding to $1 \times 10^{8} \mathrm{CFU} / \mathrm{mL}$ ), using a densitometer DEN-1 (Biosan, Riga, Latvia). Serial doubling dilutions of the tested essential oil was prepared in a 96/well microtiter plate over the range of 454.4-0.22 $\mu \mathrm{L} / \mathrm{mL}$ in inoculated Mueller-Hinton broth (MHB, HiMedia). From the last well in row $100 \mu \mathrm{L}$ of the mixture was discharged. The test was performed in a total volume of $110 \mu \mathrm{L} / \mathrm{mL}$ with a final microbial concentration $10^{6} \mathrm{CFU} / \mathrm{mL}$ per well. The plate was incubated for $24 \mathrm{~h}$ at $37{ }^{\circ} \mathrm{C}$. The same tests were 
performed simultaneously for growth control (MHB+test organism), sterility control (MHB+test oil), and positive control (MHB+gentamicin+test organism). Gentamicin was prepared in sterile water and diluted in MHB to obtain concentrations in a range of 16 to 0.016 $\mu \mathrm{g} / \mathrm{mL}$. Microbial growth was determined by adding $10 \mu$ of $0.01 \%$ resazurin (7-Hydroxy-3H-phenoxazin-3one 10-oxide, HiMedia) aqueous solution. The minimal inhibitory concentration (MIC) was defined as the lowest concentration of the samples inhibiting visible growth (blue coloured pellet on the bottom of the wells after the addition of resazurin). To determine the minimal bactericidal concentration (MBC), the broth was taken from each well without visible growth and inoculated in Mueller-Hinton agar (MHA) for $24 \mathrm{~h}$ at 37 ${ }^{\circ} \mathrm{C}$. The $\mathrm{MBC}$ was defined as the lowest sample concentration killing $99.9 \%$ of bacterial cells.

\section{Results and discussion}

\section{Chemical composition}

Hyssop essential oil is a light-yellow liquid, with a herbaceous, camphor-like odour with warm and spicy undernotes (Judžentiene, 2016). A total of 61 compounds were detected in the hyssop essential oil, among which bicyclic monoterpene ketones cispinocamphone $(43.8 \%)$ and trans-pinocamphone $(18.3 \%)$ were the most abundant, comprising $62.1 \%$. Other significant compounds were: $\beta$-pinene $(6.3 \%)$, pinocarvone $(6.1 \%)$, limonene $(3.5 \%)$, elemol $(2.5 \%)$, myrtenol (2.0\%), germacrene D $(2.0 \%)$, transcaryophyllene $(1.1 \%)$ and one unidentified compound $(1.1 \%)$. Other compounds were present in an amount less than $1.0 \%$ (Table 1). A GC-FID chromatogram of hyssop essential oil is shown in Figure 1.

Hyssop showed considerable variations in the relative content of its major components. It could be related to genotype, location, and climatic conditions (Ogunwande et al., 2011). Furthermore, high intraspecific diversity of hyssop is essential for adaptive evolution to diversify habitats (Galambosi et al., 1993, Mutu et al., 2014). According to this research, as well as research done in previous years, hyssop from Institute of Field and Vegetable Crops Novi Sad "Domaći ljubičasti" belongs to the chemotype with dominant cispinocamphone (=isopinocamphone) (Aćimović et al., 2019a, Aćimović et al., 2019b). Other than this chemotype, there are others such as: transpinocamphone, linalool, limonene, trans-pinocamphone + cis-pinocamphone and bicyclgermacrene chomotype (Ogunwande et al., 2011).

\section{Antibacterial activity}

Hyssop essential oil expressed the highest antibacterial activity against $S$. aureus (28.40 and 56.81 $\mu \mathrm{L} / \mathrm{mL}$ MIC and MBC, respectively), as well as against E. coli (ATCC 8739$)$ and B. cereus $(56.81 \mu \mathrm{L} / \mathrm{mL}$ both, MIC and MBC). Against E. coli (ATCC 10536), P. hauseri, L. monocytogenes, R. equi and L. ivanovii the MIC was $56.81 \mu \mathrm{L} / \mathrm{mL}$ of essential oil, while MBC was $113.63 \mu \mathrm{L} / \mathrm{mL}$. $S$. Enteritidis has the same value for MIC and MBC $(113.63 \mu \mathrm{L} / \mathrm{mL})$. At the same time, for E. faecalis, L. innocua and B. spirizenii the MIC was $113.63 \mu \mathrm{L} / \mathrm{mL}$ of essential oil, while MBC was 227.25 $\mu \mathrm{L} / \mathrm{mL}$. Hyssop essential oil did not express antibacterial activity against $P$. aeruginosa, Salmonella Typhimurium, $K$. aerogenes and S. epidermidis (Table 2).

H. officinalis essential oil activity varied in terms of Gram-positive and Gram-negative (Venditti et al., 2015). Similar to our study, some previous studies have observed that essential oils have more activity against Gram-positive than Gram-negative isolates. This is thought to be due to the more complex, rigid outer membrane of Gram-negative bacteria with lipopolysaccharide that limits the diffusion of hydrophobic compounds. The complex outer membrane is not present in Gram-positive bacteria, and the peptidoglycan cell wall provides less resistance against the hydrophobic compounds (Chouhan et al., 2017). It is evident that antimicrobial potential depends on the chemical composition of essential oil (Saeedi and Morteza-Semnani, 2009). For example, in the same study, $H$. officinalis ssp. officinalis (with cis-pinocamphone as the main compound) was not effective against tested bacteria, while ssp. decumbens (with linalool as the dominant compound) inhibited almost all bacteria (Mazzanti et al., 1998).

Hyssop essential oil exhibited a varying degree of inhibitory effect against two tested Salmonella strains. In our study hyssop essential oil had the efficacy against $S$. Enteritidis but had not the efficacy against $S$. Typhimurium. Previous researches conducted by Busani et al. (2004) and Musgrove et al. (2006) showed that antimicrobial resistance in Salmonella spp. is serotypically dependent. They reported that among tested Salmonella isolates, $S$. Typhimurium was the most prevalent serotype and demonstrated the greatest multiple resistance against tested antimicrobial agents. The mechanism of the antimicrobial resistance in Salmonella spp. is complex, including its resistance at the cellular level and the adaptive resistance (Penesyan et al., 2015). The mechanism of resistance at the cellular level is related to the presence of certain genes, while adaptive resistance can be explained by the ability of producing a biofilm (Corona and Martinez, 2013).

Furthermore, it is shown that $H$. officinalis oil exhibited concentration-dependent antibacterial activity (Saeedi and Morteza-Semnani, 2009). Literature review of the main essential oil components and antibacterial properties of $H$. officinalis is shown in Table 3. 
Table 1. Chemical composition of hyssop essential oil

\begin{tabular}{|c|c|c|c|c|}
\hline No & R.T. min & Compound & RI & $\%$ \\
\hline 1 & 4.611 & NI & 880 & tr \\
\hline 2 & 5.451 & $\mathrm{NI}$ & 918 & $\operatorname{tr}$ \\
\hline 3 & 5.628 & $a$-Thujene & 924 & 0.1 \\
\hline 4 & 5.820 & $a$-Pinene & 931 & 0.3 \\
\hline 5 & 6.231 & Camphene & 946 & 0.1 \\
\hline 6 & 6.916 & Sabinene & 970 & 0.9 \\
\hline 7 & 7.031 & $\beta$-Pinene & 974 & 6.3 \\
\hline 8 & 7.404 & Myrcene & 988 & 1.0 \\
\hline 9 & 7.523 & NI & 992 & 0.1 \\
\hline 10 & 8.286 & $a$-Terpinene & 1014 & 0.1 \\
\hline 11 & 8.562 & $p$-Cymene & 1022 & 0.2 \\
\hline 12 & 8.724 & Limonene & 1026 & 3.5 \\
\hline 13 & 8.794 & $\beta$-Phellandrene & 1028 & 0.3 \\
\hline 14 & 9.004 & cis- $\beta$-Ocimene & 1034 & 0.1 \\
\hline 15 & 9.381 & trans- $\beta$-Ocimene & 1044 & 0.5 \\
\hline 16 & 9.798 & $\gamma$-Terpinene & 1055 & 0.3 \\
\hline 17 & 10.108 & cis-Sabinene hydrate (IPP vs $\mathrm{OH}$ ) & 1063 & 0.4 \\
\hline 18 & 10.954 & Terpinolene & 1086 & 0.1 \\
\hline 19 & 11.377 & Linalool & 1098 & 0.6 \\
\hline 20 & 11.648 & cis-Thujone & 1104 & 0.2 \\
\hline 21 & 12.094 & trans-Thujone & 1114 & 0.1 \\
\hline 22 & 12.291 & NI & 1119 & 0.1 \\
\hline 23 & 12.976 & Nopinone & 1135 & 0.1 \\
\hline 24 & 13.022 & trans-Pinocarveol & 1136 & 0.5 \\
\hline 25 & 13.846 & $\mathrm{NI}$ & 1155 & 1.1 \\
\hline 26 & 13.994 & trans-Pinocamphone & 1159 & 18.3 \\
\hline 27 & 14.068 & Pinocarvone & 1160 & 6.1 \\
\hline 28 & 14.646 & cis-Pinocamphone & 1174 & 43.8 \\
\hline 29 & 15.089 & NI & 1185 & 0.1 \\
\hline 30 & 15.261 & $a$-Terpineol & 1188 & 0.2 \\
\hline 31 & 15.513 & Myrtenol & 1194 & 2.0 \\
\hline 32 & 15.617 & Methyl chavicol & 1197 & 0.3 \\
\hline 33 & 17.810 & NI & 1246 & 0.1 \\
\hline 34 & 20.009 & Methyl myrtenate & 1296 & 0.2 \\
\hline 35 & 21.256 & Myrtenyl acetate & 1324 & 0.2 \\
\hline 36 & 21.770 & $\delta$-Elemene & 1335 & 0.6 \\
\hline 37 & 23.858 & $\beta$-Bourbonene & 1383 & 0.5 \\
\hline 38 & 24.176 & $\beta$-Elemene & 1390 & 0.1 \\
\hline 39 & 24.747 & Methyl eugenol & 1403 & 0.2 \\
\hline 40 & 24.933 & $a$-Gurjunene & 1407 & 0.2 \\
\hline 41 & 25.345 & trans-Caryophyllene & 1417 & 1.1 \\
\hline 42 & 25.753 & $\beta$-Copaene & 1427 & 0.1 \\
\hline 43 & 26.410 & 6,9-Guaiadiene & 1443 & 0.1 \\
\hline 44 & 26.790 & $a$-Humulene & 1452 & 0.2 \\
\hline 45 & 26.930 & $\operatorname{trans}-\beta$-Farnesene & 1456 & 0.1 \\
\hline 46 & 27.101 & 9-epi-trans-Caryophyllene & 1459 & 0.7 \\
\hline 47 & 27.957 & Germacrene D & 1480 & 2.0 \\
\hline 48 & 28.267 & NI & 1488 & 0.1 \\
\hline 49 & 28.603 & Bicyclogermacrene & 1496 & 0.9 \\
\hline 50 & 29.609 & $\mathrm{NI}$ & 1521 & 0.1 \\
\hline 51 & 29.702 & $\delta$-Cadinene & 1552 & 0.1 \\
\hline 52 & 30.760 & Elemol & 1548 & 2.5 \\
\hline 53 & 31.903 & Spathulenol & 1575 & 0.7 \\
\hline 54 & 32.121 & Caryophyllene oxide & 1581 & 0.5 \\
\hline 55 & 32.928 & NI & 1600 & 0.2 \\
\hline 56 & 34.046 & $\gamma$-Eudesmol & 1629 & 0.1 \\
\hline 57 & 34.303 & NI & 1636 & 0.1 \\
\hline 58 & 34.400 & NI & 1639 & 0.1 \\
\hline 59 & 34.768 & NI & 1648 & 0.1 \\
\hline 60 & 34.887 & NI & 1651 & 0.2 \\
\hline \multirow[t]{3}{*}{61} & 41.912 & NI & 1845 & 0.1 \\
\hline & & Total & & $100 \%$ \\
\hline & & NI & & $2.5 \%$ \\
\hline
\end{tabular}




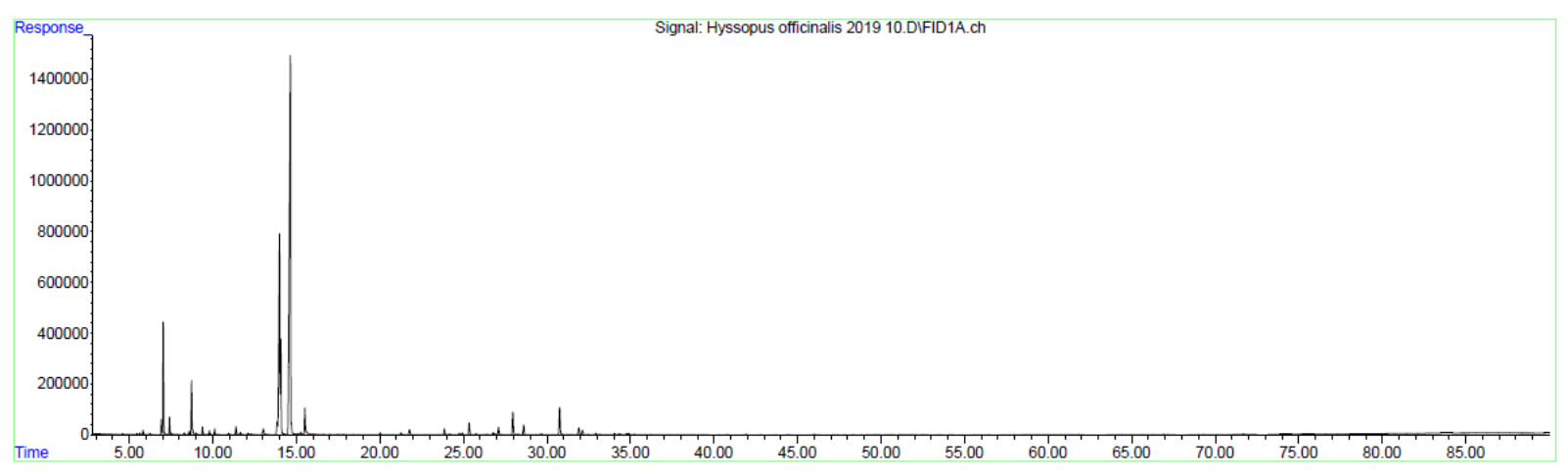

Figure 1. A GC-FID chromatogram of hyssop essential oil

Table 2. Antimicrobial properties of hyssop essential oil

\begin{tabular}{|c|c|c|c|c|c|}
\hline Gram - & $\begin{array}{c}\text { MIC } \\
(\mu \mathrm{L} / \mathrm{mL})\end{array}$ & $\begin{array}{c}\mathrm{MBC} \\
(\mu \mathrm{L} / \mathrm{mL})\end{array}$ & Gram + & $\begin{array}{c}\text { MIC } \\
(\mu \mathrm{L} / \mathrm{mL})\end{array}$ & $\begin{array}{c}\text { MBC } \\
(\mu \mathrm{L} / \mathrm{mL})\end{array}$ \\
\hline $\begin{array}{l}\text { Escherichia coli } \\
\text { ATCC } 8739\end{array}$ & 56.81 & 56.81 & $\begin{array}{l}\text { Staphylococcus aureus } \\
\text { ATCC } 25923\end{array}$ & 28.40 & 56.81 \\
\hline $\begin{array}{l}\text { Escherichia coli } \\
\text { ATCC } 10536\end{array}$ & 56.81 & 113.63 & $\begin{array}{l}\text { Bacillus cereus } \\
\text { ATCC } 11778\end{array}$ & 56.81 & 56.81 \\
\hline $\begin{array}{l}\text { Proteus hauseri } \\
\text { ATCC } 13315\end{array}$ & 56.81 & 113.63 & $\begin{array}{l}\text { Listeria monocytogenes } \\
\text { ATCC } 19111\end{array}$ & 56.81 & 113.63 \\
\hline $\begin{array}{l}\text { Salmonella Enteritidis } \\
\text { ATCC } 13076\end{array}$ & 113.63 & 113.63 & $\begin{array}{l}\text { Rhodococcus equi } \\
\text { ATCC } 6939\end{array}$ & 56.81 & 113.25 \\
\hline $\begin{array}{l}\text { Pseudomonas aeruginosa } \\
\text { ATCC } 27853\end{array}$ & $>454.50$ & $>454.50$ & $\begin{array}{l}\text { Listeria ivanovii } \\
\text { ATCC } 19119\end{array}$ & 56.81 & 113.63 \\
\hline $\begin{array}{c}\text { Salmonella } \text { Typhimurium } \\
\text { ATCC } 14028\end{array}$ & $>454.50$ & $>454.50$ & $\begin{array}{l}\text { Enterococcus faecalis } \\
\text { ATCC } 29212\end{array}$ & 113.63 & 227.25 \\
\hline \multirow[t]{3}{*}{$\begin{array}{c}\text { Klebsiella aerogenes } \\
\text { ATCC } 13048\end{array}$} & $>454.50$ & $>454.50$ & $\begin{array}{c}\text { Listeria innocua } \\
\text { ATCC } 33090\end{array}$ & 113.63 & 227.25 \\
\hline & & & $\begin{array}{l}\text { Bacillus spirizenii } \\
\text { ATCC } 6633\end{array}$ & 113.63 & 227.25 \\
\hline & & & $\begin{array}{c}\text { Staphylococcus epidermidis } \\
\text { ATCC } 12228\end{array}$ & $>454.50$ & $>454.50$ \\
\hline
\end{tabular}

The $Z$-pinocamphone as the main compound (between 39.3 and $57.3 \%$ ) was dominant in $H$. officinalis ssp. officinalis (Mazzanti et al., 1998, Kizil et al., 2010, Mahboubi et al., 2011, Aćimović et al., 2019b). The agar diffusion test did not show antimicrobial activity against Pseudomonas aeruginosa, Proteus mirabilis and Salmonella typhi. In contrast, it showed more antimicrobial activity against Klebsiella oxytoca, Escherichia coli, Enterococus faecalis and Staphylococcus aureus was more active (Mazzanti et al., 1998). Furthermore, the disk diffusion test showed antimicrobial activity against $S$. pyogenes, $S$. aureus, $C$. albicans and E. coli, but not against $P$. aeruginosa (Kizil et al., 2010). Significant antibacterial activity was noted against $S$. aureus, B. cereus and $S$. saprophyticus while Gramnegative bacteria (E. coli and $P$. aeruginosa) were less sensitive (Mahboubi et al., 2011). Furthermore, the best antimicrobial activity was noted against Bacillus cereus, followed by E. coli, S. Enteritidis, S. aureus and $S$. epidermidis (Aćimović et al., 2019b). These results indicated the possibility that antimicrobial activity does not depend on the main compound ( $Z$-pinocamphone), rather on the other compounds and their synergistic effect (Veras et al., 2012).

The dominant compound in $H$. officinalis ssp. officinalis was E-pinocamphone in $\mathrm{f}$. albus $(51.0 \%)$ and $\mathrm{f}$. ruber (28.8\%), where pink form (with almost equal amounts of both pinocamphone isomers) was more active against Gram-negative bacteria, especially against B. subtilis (Baj et al., 2018). Results also indicate synergistic and additive effects between essential oil compounds (Bassolé and Juliani, 2012, Boonyanugomol et al., 2017, Chouhan et al., 2017).

Generally, essential oils are comprised of two or three major components in relatively high concentrations $(20-95 \%)$ while other components are present in traces. The major components of EOs determine their biological properties. However, some studies have demonstrated that EOs usually have higher antibacterial activity than the mixtures of their major components, suggesting that the minor 
Table 3. Literature review of main components of essential oil and antibacterial properties of $H$. officinalis

\begin{tabular}{|c|c|c|c|c|c|c|c|c|c|c|}
\hline Accession & $\begin{array}{l}\text { H. officinalis } \\
\text { ssp. } \\
\text { decumbens }\end{array}$ & $\begin{array}{l}\text { H. officinalis } \\
\text { ssp. } \\
\text { officinalis }\end{array}$ & $\begin{array}{l}\text { H. officinalis } \\
\text { ssp. } \\
\text { angustifolius }\end{array}$ & $\begin{array}{l}\text { H. officinalis } \\
\text { ssp. } \\
\text { officinalis }\end{array}$ & $\begin{array}{l}\text { H. } \\
\text { officinalis }\end{array}$ & H. officinalis & $\begin{array}{l}H . \\
\text { officinalis } \\
\text { ssp. } \\
\text { aristatus }\end{array}$ & $\begin{array}{l}\text { H. officinalis } \\
\text { f. albus }\end{array}$ & $\begin{array}{l}\text { H. officinalis } \\
\text { f. ruber }\end{array}$ & $\begin{array}{l}\text { H.officinalis } \\
\text { ssp. officinalis }\end{array}$ \\
\hline Reference & $\begin{array}{l}\text { Mazzanti } \\
\text { et al. } 1998\end{array}$ & $\begin{array}{l}\text { Mazzanti } \\
\text { et al. } 1998\end{array}$ & $\begin{array}{l}\text { Saeedi and } \\
\text { Morteza- } \\
\text { Semnani } \\
2009\end{array}$ & $\begin{array}{l}\text { Kizil et al. } \\
2010\end{array}$ & $\begin{array}{l}\text { Mahboubi } \\
\text { et al. } 2011\end{array}$ & $\begin{array}{l}\text { Dehghanza } \\
\text { deh et al. } \\
2012\end{array}$ & $\begin{array}{l}\text { Venditti et } \\
\text { al. } 2015\end{array}$ & $\begin{array}{l}\text { Baj et al. } \\
2018\end{array}$ & $\begin{array}{l}\text { Baj et al. } \\
2018\end{array}$ & $\begin{array}{l}\text { Aćimović et } \\
\text { al. } 2019 \mathrm{~b}\end{array}$ \\
\hline $\begin{array}{l}\text { Main } \\
\text { compounds } \\
\text { in essential oil } \\
(\%)\end{array}$ & 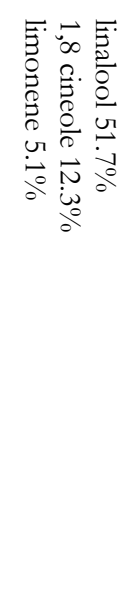 & 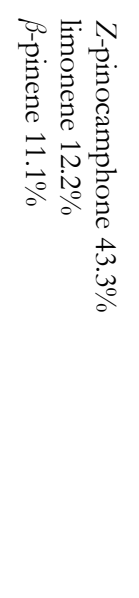 & 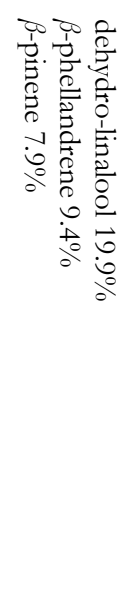 & 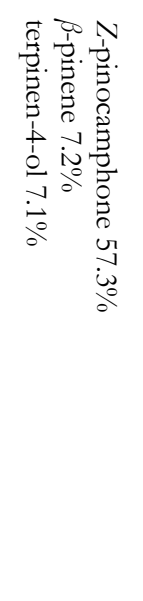 & 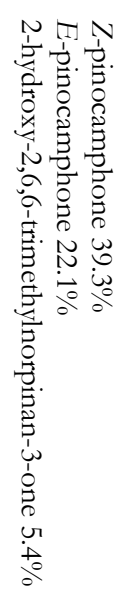 & 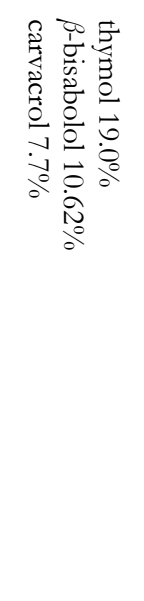 & 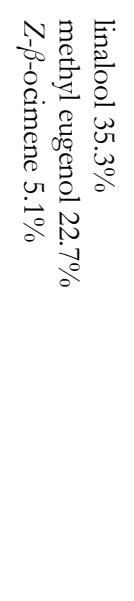 & 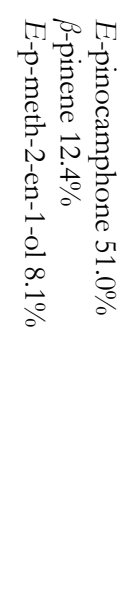 & 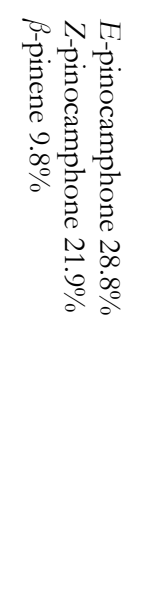 & 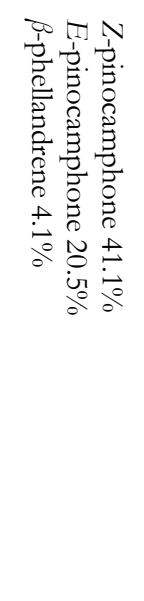 \\
\hline
\end{tabular}

\begin{tabular}{|c|c|c|c|c|c|c|c|c|c|c|}
\hline & $\begin{array}{c}\mathrm{MIC} / \\
\mathrm{MBC} \text { in } \\
\%\end{array}$ & $\begin{array}{c}\text { MIC/ } \\
\text { MBC in } \\
\%\end{array}$ & $\begin{array}{l}\text { inhibition } \\
\text { zone in } \\
\mathrm{mm} / \mathrm{MIC} \\
\text { in } \mathrm{mg} / \\
\mathrm{mL}\end{array}$ & $\begin{array}{l}\text { inhibition } \\
\text { zone in } \\
\mathrm{mm} \\
5 \mu \mathrm{L} / 10 \mu \\
\mathrm{L}\end{array}$ & $\begin{array}{l}\mathrm{MIC} / \\
\mathrm{MBC} \text { in } \\
\mathrm{mg} / \mathrm{mL}\end{array}$ & $\begin{array}{c}\text { MIC/ } \\
\text { MBC } \\
\text { inhibition } \\
\text { zone in } \\
\text { mm }\end{array}$ & $\begin{array}{l}\text { inhibitio } \\
\mathrm{n} \text { zone in } \\
\mathrm{mm}\end{array}$ & $\begin{array}{l}\mathrm{MIC} / \\
\mathrm{MBC} \text { in } \\
\mathrm{mg} / \mathrm{mL}\end{array}$ & $\begin{array}{l}\mathrm{MIC} / \\
\mathrm{MBC} \text { in } \\
\mathrm{mg} / \mathrm{mL}\end{array}$ & $\begin{array}{c}\mathrm{MIC} / \mathrm{MBC} \\
\text { in } \mu \mathrm{L} / \mathrm{mL}\end{array}$ \\
\hline B. subtilis & & & $7.8 / 0.6$ & & & & & $5.0 / 5.0$ & $0.63 / 2.5$ & \\
\hline B. cereus & & & & & $1.0 / 1.0$ & & & & & $14.2 / 28.4$ \\
\hline E. faecalis & $0.6 / 0.6$ & $>1.2 />1.2$ & & & & & 6.0 & & & $454.5 / 454.5$ \\
\hline E. amylovora & & & & & & $7.8 / 7.8$ & & & & \\
\hline E. coli & $0.3 / 0.3$ & $>1.2 />1.2$ & $-/ 1.3$ & $20.3 / 23.3$ & $4.0 / 4.0$ & & 8.9 & $5.0 / 10.0$ & $5.0 / 5.0$ & $227.3 / 227.3$ \\
\hline Klebsiella sp. & & & & & & $6.0 / 6.0$ & & & & \\
\hline K. oxytoca & $1.2 / 1.2$ & $>1.2 />1.2$ & & & & & & & & \\
\hline K. pneumoniae & & & & & & & & $5.0 / 10.0$ & $5.0 / 10.0$ & \\
\hline M. luteus & & & & & & & & $2.5 / 5.0$ & $2.5 / 5.0$ & \\
\hline P. hauseri & & & & & & & & & & $227.3 / 454.5$ \\
\hline P. mirabilis & $1.2 / 1.2$ & $>1.2 />1.2$ & & & & & & $5.0 / 10.0$ & $5.0 / 10.0$ & \\
\hline P. aeruginosa & $0.3 / 0.3$ & $>1.2 />1.2$ & $-/ 2.5$ & & $4.0 / 4.0$ & & 6.0 & $5.0 / 10.0$ & $5.0 / 10.0$ & $454.5 / 454.5$ \\
\hline S. enteritidis & & & & & & & & & & $227.3 / 227.3$ \\
\hline S. typhi & $0.6 / 0.6$ & $>1.2 />1.2$ & $7.2 / 0.6$ & & & & & & & \\
\hline S. aureus & $0.075 / 0.3$ & $>1.2 />1.2$ & $-/ 1.3$ & $18.0 / 21.7$ & $0.5 / 0.5$ & & 9.3 & $10.0 / 20.0$ & $5.0 / 10.0$ & $227.3 / 227.3$ \\
\hline S. epideridis & & & & & & & & $5.0 / 10.0$ & $2.5 / 5.0$ & $227.3 / 227.3$ \\
\hline S. saprophyticus & & & & & $1.0 / 1.0$ & & & & & \\
\hline S.pyogenes & & & & $19.0 / 23.6$ & & & & $0.63 / 1.25$ & $0.31 / 0.63$ & \\
\hline S. mutans & & & & & & & & $1.25 / 1.25$ & $0.63 / 1.25$ & \\
\hline S. pneumoniae & & & & & & & & $0.63 / 1.25$ & $0.31 / 0.63$ & \\
\hline
\end{tabular}


components are critical to the synergistic activity (Bassolé and Juliani, 2012).

Linalool (35.3-51.7\%) was the dominant compound in var. decumbens and ssp. aristatus, wild form of hyssop from Italy and France (Mazzanti et al., 1998, Venditti et al., 2015). Linalool may contribute to the greater antimicrobial activity of var. decumbens in comparison to H. officinalis (Mazzanti et al., 1998). In addition, dehydrolinalool $(19.9 \%)$ was detected in $H$. officinalis ssp. angustifolius from Iran as the main compound (Saeedi and Morteza-Semnani, 2009). However, this compound is intermedier during synthesis of linalool from pinene (Kamatou and Viljoen, 2008).

Essential oil of $H$. officinalis from Iran contains thymol (19.0\%), $\beta$-bisabolol (10.62\%) and carvacrol $(7.7 \%)$ as dominant compounds and shows high antimicrobial activities in vitro against Klebsiella sp. and Erwinia amylovora, two important plant pathogens (Dehghanzadeh et al., 2012). Furthermore, thymol is the main compound in $H$. cuspidatus from China, and this essential oil possesses contact toxicity against adult maize weevil (Sitophilus zeamais) (Li et al., 2013). Essential oil of Hyssopus sp. which contains thymol shows potential to be used as a natural pesticide.

\section{Conclusions}

The commercial variety of hyssop cv. "Domaći ljubičasti" has light-yellow essential oil, with a herbaceous, camphor-like odour with warm and spicy undernotes. GC-MS show the presence of 61 compounds, among which bicyclic monoterpene ketones cis-pinocamphone $(43.8 \%)$ and transpinocamphone $(18.3 \%)$ were the most abundant, comprising $62.1 \%$. Other significant compounds were: $\beta$ -pinene $(6.3 \%)$ and pinocarvone (6.1\%). Hyssop essential oil expressed antibacterial activity against 12 investigated bacteria (S. aureus, E. coli, B. cereus, P. hauseri, L. monocytogenes, R. equi, L. ivanovii, S. Enteritidis, E. faecalis, L. innocua and B. spirizenii). In contrast, it did not express activity against four investigated bacteria: $P$. aeruginosa, Salmonella Typhimurium, $K$. aerogenes and $S$. epidermidis. Further investigations will be focused on studying the antimicrobial activity of significant constituents of $H$. officinalis essential oil and potential synergistic or additive effects.

\section{References}

Aćimović, M. (2021). Essential oils: inhalation aromatherapy - a comprehensive review. Journal of Agronomy, Technology and Engineering Management, 4(2): 547-557.

Aćimović, M., Stanković, J., Cvetković, M., Kiprovski, B., MarjanovićJeromela, A., Rat, M., Malenčić, Đ. (2019a). Essential oil analysis of different hyssop genotypes from IFVCNS medicinal plant collection garden. Annals of Agronomy, 43, 38-45.

Aćimović, M., Todosijević, M., Varga, A., Kiprovski, B., Tešević, V., Čabarkapa, I., Sikora, V. (2019b). Bioactivity of essential oils from cultivated winter savory, sage and hyssop. Lekovite sirovine, $39,11-17$.
Baj, T., Korona-Glowniak, I., Kowalski, R., Malm, A. (2018). Chemical composition and microbiological evaluation of essential oil from Hyssopus officinalis $\mathrm{L}$. with white and pink flowers. Open Chem, 16, 317-323.

Bassolé, I.H.N., Juliani, H.R. (2012). Essential oils in combination and their antimicrobial properties. Molecules, 17, 3989-4006.

Boonyanugomol, W., Kraisriwattana, K., Rukseree, K., Boonsam, K., Narachai, P. (2017). In vitro synergistic antibacterial activity of the essential oil from Zingiber cassumunar Roxb against extensively drug-resistant Acinetobacter baumannii strains. Journal of Infection and Public Health, 10, 586-592.

Busani, L., Graziani, C., Battisti, A., Franco, A., Ricci, A., Vio, D., Digiannatale, E., Paterlini, F., DIncau, M., Owczarek, S., Caprioli, A., Luzzi, I. (2004). Antibiotic resistance in Salmonella enterica serotypes Typhimurium, Enteritidis and Infantis from human infections, food stuffs and farm animals min Italy. Epidemiology and Infection, 132, 245-251.

Chouhan, S., Sharma K., Guleria S. (2017). Antimicrobial activity of some essential oils - present status and future perspectives. Medicines, 4, 58; https://doi.org/10.3390/medicines4030058

Corona, F., Martinez J.L. (2013). Phenotypic resistance to antibiotics. Antibiotics, 2, 237-255.

Dehghanzadeh, N., Ketabchi, S., Alizadeh, A. (2012). Essential oil composition and antibacterial activity of Hyssopus officinalis L. grown in Iran. Asian Journal of Experimental Biological Sciences, 3 767-771.

Fathiazad, F., Hamedeyazdan, S. (2011). A review on Hyssopus officinalis L.: Composition and biological activities. African Journal of Pharmacy and Pharmacology 5, 1959-1966.

Fraternale, D., Ricci, D., Epifano, F., Curini, M. (2004). Composition and antifungal activity of two essential oils of hyssop (Hyssopus officinalis L.). Journal of Essential Oil Research, 16, 617-622.

Galambosi, B., Svoboda, K., Deans, S., Hethelyi, E. (1993). Agronomical and phytochemical investigation of Hyssopus officinalis. Agricultural Science in Finland, 2, 293-302.

Gorunović, M.S., Bogavac, P.M., Chalchat, J.C., Chabard, J.L. (1995). Essential oil of Hyssopus officinalis L., Lamiaceae of Montenegro origin. Journal of Essential Oil Research, 7, 39-43.

Hamida, N.B., Martinez-Diaz, R.A., Hela, M., Msaada, K., Ouerghi, Z., Andres, M.F., Gonzalez-Coloma, A. (2020). Effect of salinity on the antiparasitic activity of hyssop essential oil. Journal of Essential Oil Research, 32, 69-78. https:// doi.org/10.1080/10412905.2019.1656677

Jahantigh, O., Najafi, F., Badi, H.N., Khavari-Nejad, R.A., Sanjarian, F. (2016). Essential oil composition of hyssop (Hyssopus officinalis L.) under salt tress at flowering stage. Journal of Essential Oil Research, $28, \quad 458-464$. ht t p : / / dx.doi.org/10.1080/10412905.2016.1153001

Josifović, M. (1975). Flora SR Srbije, Tom 6. Srpska akademija nauke i umetnosti, Beograd.

Judžentiene, A. (2016). Hyssop (Hyssopus officinalis L.) oils. Preedy V.R. (ed): Essential oils in food preservation, flavor and safety. Academic Press, pp: 471-479. https://doi.org/10.1016/B978-012-416641-7.00053-5.

Kamatou, G.P.P., Viljoen, A.M. (2008). Linalool - a review of a biologically active compound of commercial importance. Natural Product Communications, 3, 1183-1192.

Kizil, S., Hasimi, N., Tolan, V., Kilinc, E., Karatas, H. (2010). Chemical composition, antimicrobial and antioxidant activities of hyssop (Hyssopus officinalis L.) essential oil. Notulae Botanicae Horti Agrobotanici Cluj-Napoca, 38, 99-103.

Kizil, S., Toncer, O., Ipek, A., Arslan, N., Saglam, S., Khawar, K.M. (2008). Blooming stages of Turkish hyssop (Hyssopus officinalis L.) affect essential oil composition. Acta Agriculturae Scandinavica Section B - Soil and Plant Science, 58, 273-279. Doi: 10.1080/09064710701647297

Li, H.T., Zhao, N.N., Yang, K., Liu, Z.L., Wang, Q. (2013). Chemica composition and toxicities of the essential oil derived from Hyssopus cuspidatus flowering aerial parts against Sitophilus zeamais and Heterodera avenae. Journal of Medicinal Plant Research, 7, 343-348. DOI: $10.5897 /$ JMPR12.475. 
Mahboubi, M., Haghi, G., Kazempour, N. (2011). Antimicrobial activity and chemical composition of Hyssopus officinalis L. essential oil. Journal of Biologically Active Products from Nature, 1, 132 137.

Mazzanti, G., Battinelli, L., Salvatore, G. (1998). Antimicrobial properties of the linalol-rich essential oil of Hyssopus officinalis L. var decumbens (Lamiaceae). Flavour and Fragrance Journal, 13, 289294.

Moro, A., Zalacain, A., de Mendoza, J.H., Carmona, M. (2011). Effects of agronomic practices on volatile composition of Hyssopus officinalis L. essential oils. Molecules, 16, 4131-4139. Doi: 10.3390/molecules16054131.

Musgrove, M.T., Jones, D.R., Northcutt, J.K., Cox, N.A., Harrison, M.A., Fedorka-Cray, P.J., Ladely, S.R. (2006). Antimicrobial resistance in Salmonella and Escherichia coli isolated from commercial shell eggs. Poultry Science, 85, 1665-1669.

Mutu, A., Clapco, S., Martea, R., Port, A., Gille, E., Duca, M. (2014). Intraspecific genetic variability of Hyssopus officinalis L. Anale Stiintifice ale Universitati "Alexandru Ioan Cuza", Sectiunea Genetica si Biologie Moleculara, 15, 1-8.

Nanova, Z., Slavova, Y., Nenkova, D., Ivanova, I. (2007). Microclonal propagation of hyssop (Hyssopus officinalis L.). Bulgarian Journal of Agricultural Science, 13, 213-219.

Nemeth-Zambori, E., Rajhart, P., Inotai, K. (2017). Effect of genotype and age on essential oil and total phenolics in hyssop (Hyssopus officinalis L.). Journal of Applied Botany and Food Quality, 90, 25-30.

Ogunwande, I., Flamini, G., Alese, O., Cioni, P., Ogundajo, A., Setzer, W. (2011). A new chemical form of essential oil of Hyssopus officinalis L. (Lamiaceae) from Nigeria. International Journal of Biological and Chemical Sciences, 5, 46-55.

Ozer, H., Sahin, F., Kilic, H., Gulluce, M. (2005). Essential oil composition of Hyssopus officinalis L. subsp. angustifolius (Bieb.) Arcangeli from Turkey. Flavour and Fragrance Journal, 20, 42-44.
Penesyan, A., Gillings, M., Paulsen, I.T. (2015). Antibiotic discovery: combatting bacterial resistance in cells and in biofilm communities. Molecules, 20, 5286-5298.

Piccaglia, R., Pace, L., Tammaro, F. (1999). Characterization of essential oils from three Italian ecotypes of hyssop [Hyssopus officinalis L. ssp. aristatus (Gordon) Briq.]. Journal of Essential Oil Research, 11, 693-699.

Saeedi, M., Morteza-Semnani, K. (2009). Chemical composition and antimicrobial activity of essential oil of Hyssopus angustifolius M.B. Journal of Essential Oil Bearing Plants, 12, 111-119.

Said-Al Ahl, H., Abbas, Z., Sabra, A., Tkachenko, K. (2015). Essential oil composition of Hyssopus officinalis L. cultivated in Egypt. International Journal of Plant Science and Ecology, 1, 49-53.

Tahir, M, Khushtar, M, Fahad, M, Rahman, A. (2018). Phytochemistry and pharmacological profile of traditionally used medicinal plant Hyssop (Hyssopus officinalis L.). Journal of Applied Pharmaceutical Sciences, 8, 132-140.

Tucakov, J. (2006). Lečenje biljem: fitoterapija [Healing with plants: phytotherapy]. Rad, Beograd.

Venditti, A., Bianco, A., Frezza, C., Conti, F., Bini, L.M., Giuliani, C., Bramucci, M., Quassinti, L., Damiano, S., Lupidi, G., Beghelli, D., Caterbi, S., Peterelli, D., Vitali, L.A., Papa, F., Caprioli, G., Maggi, F. (2015). Essential oil composition, polar compounds, glandular trichomes and biological activity of Hyssopus officinalis subsp. aristatus (Godr.) Nyman from central Italy. Industrial Crops and Products, 77, 353-363.

Veras, H.N.H., Rodrigues, F.F.G., Colares, A.V., Menezes, I.R.A., Coutinho, H.D.M., Botelho, M.A., Costa, J.G.M. (2012). Synergistic antibiotic activity of volatile compounds from the essential oil of Lippia sidoides and thymol. Fitoterapia, 83, 508512.

Zawislak, G. (2013). Morphological characters of Hyssopus officinalis L. and chemical composition of its essential oil. Modern Phytomorphology, 4, 93-95.

\section{Hemijski sastav etarskog ulja miloduha kultivara „Domaći ljubičasti“ i ocena antimikrobne aktivnosti}

\section{Milica Aćimović · Ana Varga · Mirjana Cvetković · Pezo Lato · Biljana Lončar · Maja Ignjatov · Tijana Zeremski}

Sažetak: Miloduh (Hyssopus officinalis L., Lamiaceae) je višegodišnji žbun ili polužbun aromatičnog i oštrog mirisa zbog čega se koristi kao začinska biljka. Takođe je i ukrasna i medonosna biljka, ali i tradicionalni lek za bolesti organa za disanje i kod poremećaja varenja. Miloduh je cenjena eterično-aromatična biljka, a etarsko ulje se koristi u farmaceutskoj, parfimerijskoj i kozmetičkoj industriji kao i aromaterapiji. Cilj ovog istraživanja je bio da se determiniše hemijski sastav etarskog ulja miloduha kultivara „Domaći ljubičasti“ gajenog u Srbiji, i da se ispita antimikrobna aktivnost na 16 bakterija, uglavnom patogena u prehrambenoj industriji. Ukupno 61 komponenta je detektovana u etarskom ulju, pri čemu su monoterpenski ketoni cis-pinokamfon (43.8\%) i trans-pinokamfon (18.3\%) bili najzastupljeniji, čineći $62.1 \%$, a potom slede $\beta$-pinen i pinokarvon. Etarsko ulje miloduha je ispoljilo antibakterijsku aktivnost na: Staphylococcus aureus, Escherichia coli, Bacillus cereus, Proteus hauseri, Listeria monocytogenes, Rhodococcus equi, Listeria ivanovii, Salmonella Enteritidis, Enterococcus faecalis, Listeria innocua i Bacillus spizizenii, dok nije delovalo na Pseudomonas aeruginosa, Salmonella Typhimurium, Klebsiella aerogenes i Staphylococcus epidermidis.

Ključne reči: antibakterijska aktivnost, etarsko ulje, Hyssopus officinalis, miloduh, pinokamfon

This article is distributed under the terms of the Creative Commons Attribution 4.0 International License (http://creativecommons.org/licenses/by/4.0), which permits unrestricted use, distribution and reproduction in any medium, provided you give appropriate credit to the original author(s) and the source, provide a link to the Creative Commons license, and indicate if changes were made. 